Discrete Comput Geom 28:491-494 (2002)

DOI: $10.1007 / \mathrm{s} 00454-002-2895-0$

\title{
The Number of Directions Determined by Points in the Three-Dimensional Euclidean Space*
}

\author{
Aart Blokhuis ${ }^{1}$ and Ákos Seress ${ }^{2}$ \\ ${ }^{1}$ Eindhoven University of Technology, \\ 5600 MB Eindhoven, The Netherlands \\ aart@win.tue.nl \\ ${ }^{2}$ Department of Mathematics, The Ohio State University, \\ Columbus, OH 43210, USA \\ akos@math.ohio-state.edu
}

\begin{abstract}
Let $X$ be a set of $n$ points in the three-dimensional Euclidean space such that no three points in $X$ are on the same line and there is no plane containing all points in $X$. An old conjecture states that pairs of points in $X$ determine at least $2 n-3$ directions. We prove the weaker result that $X$ determines at least $1.75 n-2$ directions.
\end{abstract}

\section{Introduction}

Let $X$ be a point set in the three-dimensional Euclidean space $\mathbb{R}^{3}$. We say that $X$ is in general position if no three points in $X$ are on the same line and there is no plane containing all points in $X$. The directions determined by $X$ are the directions of the lines going through pairs of points in $X$. We say that a direction $\delta$ determined by $X$ has multiplicity $k$ if there are exactly $k$ pairs of points in $X$ such that the line through these pairs of points is in direction $\delta$. This note is about the following conjecture.

Conjecture 1. If $X \subseteq \mathbb{R}^{3}$ is in general position and $|X|=n$, then $X$ determines at least $2 n-3$ directions.

The first version of the conjecture in print (in the form that $X$ is non-planar, but more than two points of $X$ may be on the same line) seems to appear in [Sc]. This problem is similar to the problem of Erdôs that asks for the minimal number of lines determined

* The research by the second author was partially supported by the NSF. 
by $n$ non-collinear points (see [St]). As far as we know, Erdôs's problem is not solved in dimensions greater than two.

Finding the minimal number of directions determined by $n$ points on the plane, when no three of them are collinear, is easy.

Proposition 2. If $X$ is a set of $n$ points on the plane such that no three points are on the same line, then $X$ determines at least $n$ directions.

Proof. Let $p_{1}, p_{2}, p_{3} \in X$ be three consecutive points among the points of $X$ which are on the boundary of the convex hull of $X$. The lines through $p_{2}$ determine $n-1$ directions, and the direction of the line through $p_{1}$ and $p_{3}$ is different from these.

The extremal configurations for Proposition 2 were determined by Jamison [Ja3]. Proposition 2 has the following non-trivial strengthening.

Theorem 3 [Un]. If $X$ is a set of $n$ points on the plane such that not all of them are on the same line, then $X$ determines at least $2\lfloor n / 2\rfloor$ directions.

We cannot prove Conjecture 1, but we shall prove the following two theorems.

Theorem 4. If $X \subseteq \mathbb{R}^{3}$ is a set of $n$ points in general position and there is a direction determined by $X$ of multiplicity $k$, then $X$ determines at least $2 n-2-k$ directions.

Theorem 5. If $X \subseteq \mathbb{R}^{3}$ is a set of $n$ points in general position, then it determines a direction with multiplicity at most $n / 4$.

Corollary 6. If $X \subseteq \mathbb{R}^{3}$ is a set of $n$ points in general position, then it determines at least $1.75 n-2$ directions.

We finish this Introduction with three more conjectures. The last two of these are similar to Conjecture 6.3 of [Ja2].

Conjecture 7. If $X \subseteq \mathbb{R}^{3}$ is in general position, then it determines a direction of multiplicity at most 2 .

Conjecture 8. If $X \subseteq \mathbb{R}^{3}$ is a set of $n$ points which are not all in the same plane, then $X$ determines at least $2 n-5$ directions.

Conjecture 9. If $X \subseteq \mathbb{R}^{d}, d \geq 2$, is a set of $n$ points which are not all in the same hyperplane and no three points of $X$ are on the same line, then $X$ determines at least $(d-1) n-d(d-2)$ directions. 


\section{Examples}

Conjecture 1, if true, is sharp. We know of one infinite series and four sporadic extremal configurations. Of course, any affine image of these configurations is also a suitable example.

(1) Let $n$ be even and $X_{1, n}=\left\{p_{1}, \ldots, p_{n}\right\}$ such that

(i) $p_{1}, \ldots, p_{n-2}$ are the vertices of a regular $(n-2)$-gon $Y$;

(ii) $p_{n-1}, p_{n}$ are on the line orthogonal to the plane of $Y$ which goes through the center $o$ of $Y$; and

(iii) $o$ is the middle point of the segment between $p_{n-1}$ and $p_{n}$.

(2) $X_{2}=\{(1,1,1),(1,1,-1),(1,-1,1),(-1,1,1),(1,-1,-1),(-1,1,-1)$, $(-1,-1,1),(-1,-1,-1)\}$ (i.e., the vertex set of a cube).

(3) $X_{3}=X_{2} \cup\{(2,0,0),(-2,0,0)\}$

(4) $X_{4}=X_{3} \cup\{(0,2,0),(0,-2,0)\}\left(X_{4}\right.$ also can be obtained as an affine image of the mid-points of the edges of a cube).

(5) $X_{5}=X_{4} \cup\{(0,0,2),(0,0,-2)\}\left(X_{5}\right.$ also can be obtained as an affine image of the symmetric difference of the vertex sets of the unit cube and its translate by the vector $(1,1,1))$.

Theorem 3 is sharp. Four infinite families and more than 100 sporadic extremal configurations are listed in [Ja1] and [JH].

Conjecture 8, if true, is also sharp. Examples (1)-(5) above are centrally symmetric, and adding the center we obtain an extremal configuration for Conjecture 8. Also, any centrally symmetric configuration from the lists in [Ja1] and [JH] can be augmented to a double cone $X$ as in example (1), which determines $2|X|-5$ directions. The same augmentation procedure can be continued to obtain higher-dimensional configurations which determine only a few directions (see Theorem 6.2 of [Ja2]).

\section{Proof of the Theorems}

Proof of Theorem 4. Let $X \subseteq \mathbb{R}^{3}$ be a set of $n$ points in general position, and let $\delta$ be a direction determined by $X$ of multiplicity $k$. Let $Y$ be the projection of $X$ onto a plane orthogonal to $\delta$. Then $Y$ consists of $n-k$ points, and $k$ of these are "double" (i.e., the projection of two points of $X$ ). Let $p$ be one of these double points, and let $L_{1}, \ldots, L_{m}$ be the lines determined by $Y$ that go through $p$. Let $k_{i}$ denote the number of points of $X$ whose projection is on $L_{i}$, but the projection is different from $p$. Since the lines $L_{i}$ contain the projections of all points of $X$, we have

$$
\sum_{i=1}^{m} k_{i}=n-2
$$

Clearly, if for two pairs of points in $X$ the directions determined by their projections in $Y$ are different, then the directions determined by them in $X$ are also different.

Since $X$ is not planar, the points in $Y$ are not all on the same line. Therefore, by Theorem 3, the points in $Y$ determine at least $n-k-1$ directions. At least $n-k-1-m$ 
of these are not the directions of the lines $L_{1}, \ldots, L_{m}$. Our next observation is that by Proposition 2, the $k_{i}+2$ points of $X$ whose projections are on line $L_{i}$ determine at least $k_{i}+1$ directions different from $\delta$. Finally, counting also the direction $\delta$ and using (1), we obtain that $X$ determines at least

$$
(n-k-1-m)+\sum_{i=1}^{m}\left(k_{i}+1\right)+1=2 n-2-k
$$

directions.

Proof of Theorem 5. Let $X \subseteq \mathbb{R}^{3}$ be a set of $n$ points in general position, and let $\Delta=\left\{\delta_{1}, \ldots, \delta_{m}\right\}$ be the set of directions determined by $X$, considered as a subset of the projective plane. Since $X$ is not planar, not all points of $\Delta$ are on the same line. Therefore, by the Gallai-Sylvester theorem (see [St]) there are $\delta_{i}, \delta_{j} \in \Delta$ such that no other points of $\Delta$ are on the line determined by $\delta_{i}$ and $\delta_{j}$. Let $k_{i}$ and $k_{j}$ denote the multiplicity of the direction $\delta_{i}$ and $\delta_{j}$ in $X$, respectively. Let $X_{i}$ be the subset of $2 k_{i}$ points in $X$ which is the union of the $k_{i}$ pairs determining the direction $\delta_{i}$. We define the subset $X_{j}$ of $2 k_{j}$ points analogously, using the pairs of points determining the direction $\delta_{j}$. The sets $X_{i}$ and $X_{j}$ are disjoint, since if $p, q \in X$ determined the direction $\delta_{i}$ and $p, r \in X$ determined the direction $\delta_{j}$ then $q$ and $r$ would determine a direction which is on the line through $\delta_{i}$ and $\delta_{j}$. Hence $2 k_{i}+2 k_{j} \leq n$ and so at least one of $k_{i}, k_{j}$ must be no greater than $n / 4$.

\section{References}

[Ja1] Jamison, R. E. Planar configurations which determine few slopes. Geom. Dedicata 16 (1984), 17-34.

[Ja2] Jamison, R. E. A survey of the slope problem. In: Discrete Geometry and Convexity. Annals of the New York Academy of Sciences 440. 1985, pp. 34-51.

[Ja3] Jamison, R. E. Few slopes without collinearity. Discrete Math. 60 (1986), 199-206.

[JH] Jamison, R. E.; Hill, D. A catalogue of sporadic slope-critical configurations. Proceedings of the Fourteenth Southeastern Conference on Combinatorics, Graph Theory and Computing (Boca Raton, FL, 1983). Congressus Numererantium, 40. Utilitas Math., Winnipeg, 1983, pp. 101-125.

[Sc] Scott, P. R. On the sets of directions determined by $n$ points. Amer. Math. Monthly 77 (1970), 502-505.

[St] Steinberg, R. Solution to Problem 4065. Amer. Math. Monthly 51 (1944), 169-171.

[Un] Ungar, P. $2 N$ noncollinear points determine at least $2 N$ directions. J. Combin. Theory Ser. A 33 (1982), 343-347.

Received February 9, 2001, and in revised form July 11, 2001. Online publication October 29, 2002. 